Review

\title{
Right ventricular outflow tract endocardial unipolar substrate mapping: implications in risk stratification of Brugada syndrome
}

\author{
Konstantinos P. Letsas ${ }^{1, *}$, Konstantinos Vlachos ${ }^{1}$, Michael Efremidis ${ }^{1}$, Stylianos Dragasis ${ }^{1}$, \\ Panagiotis Korantzopoulos ${ }^{2}$, Gary Tse ${ }^{3,4}$, Tong Liu ${ }^{4}$, George Bazoukis ${ }^{5,6}$, \\ Panagioula Niarchou ${ }^{7}$, Efstathia Prappa ${ }^{7}$, Frederic Sacher ${ }^{8}$, Meleze Hocini $^{8}$, \\ Adrian Baranchuk ${ }^{9}$
}

${ }^{1}$ Arrhythmia Unit, Onassis Cardiac Surgery Center, 17674 Athens, Greece

${ }^{2}$ Department of Cardiology, University of Ioannina Medical School, 45110 Ioannina, Greece

${ }^{3}$ Kent and Medway Medical School, CT2 7FS Canterbury, UK

${ }^{4}$ Department of Cardiology, Tianjin Key Laboratory of Ionic-Molecular Function of Cardiovascular disease, Tianjin Institute of Cardiology, Second Hospital of Tianjin Medical University, 300211 Tianjin, China

${ }^{5}$ Department of Cardiology, Medical School, University of Nicosia, 2408 Nicosia, Cyprus

${ }^{6}$ Department of Cardiology, Larnaca General Hospital, 7036 Larnaca, Cyprus

${ }^{7}$ Second Department of Cardiology, Evangelismos General Hospital of Athens, 10676 Athens, Greece

${ }^{8}$ Electrophysiology and Ablation Unit, Bordeaux University Hospital (CHU), 33600 Bordeaux, France

${ }^{9}$ Division of Cardiology, Queen's University, Kingston General Hospital, Kingston, K7K Ontario, Canada

*Correspondence: k.letsas@gmail.com; letsas@ocsc.gr (Konstantinos P. Letsas)

Academic Editor: Peter A. McCullough

Submitted: 12 October 2021 Revised: 29 December 2021 Accepted: 8 January 2022 Published: 25 January 2022

\begin{abstract}
Brugada syndrome $(\mathrm{BrS})$ is a complex arrhythmogenic disease displaying electrical and micro-structural abnormalities mainly located at the epicardium of the right ventricular outflow tract (RVOT). It is well-known that fibrosis, fatty infiltration, inflammation and reduced gap junction expression have been demonstrated at the epicardial anterior aspect of the RVOT providing the arrhythmogenic substrate for ventricular arrhythmic events in BrS. A number of models have been proposed for the risk stratification of patients with BrS. Endocardial unipolar electroanatomical mapping is an emerging tool that has been reintroduced to identify and quantify epicardial electrical abnormalities. Interestingly, current findings correlate the presence of large-sized endocardial unipolar electroanatomical abnormalities with either ventricular fibrillation inducibility during programmed ventricular stimulation or symptom status. This review aims to present existing data about the role of endocardial unipolar electroanatomical mapping for the identification of RVOT epicardial abnormalities as well as its potential clinical implications in risk stratification of $\mathrm{BrS}$.
\end{abstract}

Keywords: Brugada syndrome; Electroanatomical mapping; Risk stratification

\section{Introduction}

Brugada syndrome (BrS), a complex arrhythmogenic disease displaying electrical and micro-structural abnormalities mainly located at the epicardium of the right ventricular outflow tract (RVOT), is characterized by J-point elevation in the right precordial leads on the 12-lead electrocardiogram (ECG) and an increased risk of sudden cardiac death (SCD) due to ventricular fibrillation (VF) [1-5]. $\mathrm{BrS}$ is an inherited disease that is usually transmitted in an autosomal dominant manner [6]. The BrS related mutations are mainly associated with encoding of sodium channel, calcium channels and potassium channels [7]. The most important mutation is the SCN5A, encoding the sodium channel $\alpha$-subunit, account for 20 to $30 \%$ of patients with $\operatorname{BrS}[7,8]$. Specifically, BrS has been associated with reduced INa and loss-of-function SCN5A gene mutations. Regarding the pathophysiological basis of $\mathrm{BrS}$, abnormal depolarization, abnormal repolarization, and current-load- mismatch are the three hypotheses that have been proposed in the literature $[9,10]$.

Fibrosis, fatty infiltration, inflammation and reduced gap junction expression have been demonstrated at the anterior aspect of the RVOT epicardium providing the arrhythmogenic substrate for sustained VF in $\operatorname{BrS}[4,5,11,12]$. Epicardial substrate catheter ablation has been associated with electrocardiogram normalization and ventricular arrhythmia non-inducibility in patients with BrS supporting the current consideration of an epicardial disease [13-15]. Endocardial unipolar electroanatomical mapping (EAM) is an emerging tool that has been reintroduced into clinical practice for the identification and quantification of epicardial electrical abnormalities in specific cardiomyopathies with several clinical implications, including risk stratification for sudden cardiac death [16]. Risk stratification for BrS patients, and especially of asymptomatic ones, remains challenging [17]. Due to certain limitations of the current 
risk factors predictors, including programmed ventricular stimulation (PVS), multiparametric scores have been established for risk stratification [18,19]. This review article questions the accuracy of endocardial unipolar EAM for the identification of RVOT epicardial electrical abnormalities as well as its potential clinical implications in risk stratification of $\mathrm{BrS}$.

\section{The arrhythmogenic substrate of Brugada syndrome is related to structural abnormalities}

Accumulating data suggest that mild structural abnormalities including inflammation, fatty infiltration, and fibrosis, mainly located at the epicardial aspect of the RVOT, provide the arrhythmia substrate in BrS. Lymphocytic myocarditis has been detected in endomyocardial biopsy samples in $77 \%$ of patients with BrS without structural abnormalities assessed by imaging techniques [3]. Nademanee et al. [4] studied autoptic samples from BrS patients and showed an epicardial to endocardial gradient of collagen deposition, indicating a progression of the pathological process from the epicardium to endocardium, which correlated with low expression of Connexin 43 (Cx43). Interestingly, BrS has been associated with increased collagen content throughout the right and left ventricular myocardium [12]. Miles et al. [12] evaluated 28 whole hearts from consecutive SCD cases attributed to $\mathrm{BrS}$ and 29 hearts from a control group comprised of non-cardiac deaths. Cardiac tissue from $\mathrm{BrS}$ decedents displayed a higher amount of collagen in relation to control subjects. The highest collagen accumulation was observed at the epicardial RVOT (geometric mean area 23.7\%) [12]. Pieroni et al. [11] performed EAM-guided endomyocardial biopsies demonstrating histopathological abnormalities, including fibrosis and lymphomononuclear infiltrates in the majority of cases. EAM abnormalities manifested as low voltage unipolar and bipolar areas correlated with myocardial inflammation. Zumhagen et al. [20] showed that 55\% of BrS patients and normal imaging of the right ventricle (RV) display histopathologic abnormalities (fibrosis, inflammation, and fatty tissue). A similar study identified fatty tissue infiltration, interstitial fibrosis, and lymphocyte infiltration in $52 \%$ of BrS with inducible VF [21].

Imaging studies have also provided evidence that subtle cardiac structural abnormalities exist in BrS patients. Computed tomography studies demonstrated wall motion abnormalities within the RVOT in most BrS patients with cardiac arrest [22]. Mild reduction of RVOT ejection fraction, dilatation of the RV inflow tract diameter, and increased RV end-systolic volume have been reported in BrS patients by cardiac magnetic resonance imaging (MRI) $[23,24]$. Electromechanical substrate abnormalities during ajmaline challenge including an increase of the electrical substrate in combination with worsening of the mechanical function of the RV, particularly in the anterior free wall of RVOT, have been recently demonstrated [25].

EAM studies have consistently demonstrated the presence of abnormal substrate at the epicardium of the RVOT. In Nademanee's seminal report, abnormal electrograms characterized by low voltage $(<1 \mathrm{mV})$, prolonged duration (>120 ms), and fractionated late potentials clustering in the anterior aspect of the RVOT epicardium were demonstrated [26]. Following drug challenge with ajmaline, a 2-fold increase of these areas has been noticed [13]. Of note, the arrhythmogenic substrate detected during electroanatomical mapping has been correlated with fibrosis in vivo tissue samples [4]. The presence of a localized region in the anterior aspect of the epicardial RVOT with conduction slowing evidenced by prolonged electrogram duration (78.79 \pm $19.87 \mathrm{~ms}$ vs. $58.93 \pm 10.11 \mathrm{~ms}$ in the epicardial right ventricle, and $59.87 \pm 12.61 \mathrm{~ms}$ in the endocardial RVOT) with variable low voltage $(0.97 \pm 0.48 \mathrm{mV}$; median scar area $19.8 \pm 25.9 \mathrm{~cm}^{2}$ ) has been demonstrated in a different study [27]. In a high-density endocardial EAM study, we recorded fractionated potentials of mean duration 94.7 $\pm 21.2 \mathrm{~ms}$ in all BrS patients mainly sited within the low bipolar voltage areas at the free wall of the RVOT [28]. In addition, the mean RVOT activation time (latest endocardial activation at the sub-pulmonary valve RVOT regions) was significantly prolonged in $\mathrm{BrS}$ patients compared to the control group $(86.4 \pm 16.5 \mathrm{~ms}$ vs. $63.4 \pm 9.7 \mathrm{~ms})$. Isochronal mapping demonstrated lines of conduction slowing predominantly at the free wall of the RVOT. Interestingly, Pannone et al. [15] found a similar duration for lowfrequency potentials of $87.1 \mathrm{~ms} \pm 23.1$ at the epicardium.

Lambiase et al. [29], using high-resolution noncontact endocardial mapping, have demonstrated significant regional conduction delays, reduction in the activation gradient, and formation of lines of functional conduction block at the anterolateral free wall of the RVOT compared to the body and the apex of the RV. Postema et al. [30] have shown conduction slowing and abnormal conduction velocity restitution in the RV in BrS. These data are consistent with the depolarization hypothesis regarding the pathophysiology of $\mathrm{BrS}$ [17]. The cardiac intercalated disc is the host of a protein interacting network, called "the connexome", where different molecules including desmosomes, fascia adherence junctions, gap junctions, and voltage-gated sodium channels interact together to control excitability, electrical coupling, and intercellular adhesion in the heart. Although $\mathrm{BrS}$ is considered a primary electrical disease, microstructural abnormalities may enhance the electrical heterogeneity by affecting any of the "connexome" components [31,32]. Sodium channel activity could be affected by the disruption of any "connexome" components. Cx43 expression that is required for Nav1.5 stability in the intercalated disk membrane is impaired in BrS [4]. 


\section{Definition of the abnormal endocardial unipolar substrate of the right ventricular outflow tract}

The wide "field of view" of endocardial unipolar EAM allows identifying tissue abnormalities deeper in the midmyocardium and epicardium. Previous studies, using different methodologies (either using the 95\% confidence interval in healthy patients or direct comparison of adjacent endocardial and epicardial points), have proposed different endocardial unipolar voltage cut-off values for the identification of abnormal epicardial abnormalities of the RVOT. Polin et al. [33] have initially suggested the 5.5 $\mathrm{mV}$ cut-off criterion by using the $95 \%$ confidence interval in healthy control patients. Of note, mapping was performed using a 4-mm-tip electrode ablation catheter (2mm-ring electrode separated by $1-\mathrm{mm}$ spacing), and the sampling points ranged between 105 and 164. In a highdensity EAM study (1019.1 \pm 171.7 points $)$ performed with a multi-electrode catheter ( $2 \mathrm{~mm}$-tip, 2-8-2 mm interelectrode spacing) in subjects with idiopathic RVOT ventricular arrhythmias and negative cardiac MRI, we have shown that the mean amplitude of unipolar electrograms within the RVOT segments was $7.9 \pm 0.7 \mathrm{mV}$, with $95 \%$ of the recorded unipolar signals having an amplitude $>4$ $\mathrm{mV}$ [28]. In a combined endocardial-epicardial mapping study (3.5-mm-tip electrode ablation catheter, separated by 1-mm spacing from a 2-mm ring electrode) where each endocardial mapping point was matched to the corresponding nearest epicardial point, a $4.4 \mathrm{mV}$ unipolar voltage cutoff value has been suggested for detection of epicardial abnormalities [34]. In an elegant study comparing opposing endocardial and epicardial electrograms with a $3.5 \mathrm{~mm}$-tip mapping catheter, in areas with normal endocardial bipolar voltage, the optimal endocardial unipolar voltage cutoff value for the detection of an epicardial scar not caused by fat was $3.9 \mathrm{mV}$ [35]. The optimal endocardial unipolar voltage cut-off value to detect fragmented electrogram and late potentials at the epicardium was $3.7 \mathrm{mV}$. Of note, the latter study provides epicardial fat information as detected by computed tomography (CT), which displays significant limitations during epicardial mapping. Chrispin et al. [36] have shown that when unipolar endocardial mapping is performed with a multi-electrode catheter (1-mm-tip, 4-4$4 \mathrm{~mm}$ interelectrode spacing) instead of the conventional 3.5-mm-tip mapping/ablation catheters, a cut-off of $3.3 \mathrm{mV}$ should be used to identify the presence of epicardial RV free wall abnormalities. In structurally normal hearts, the pointby-point mapping performed using a $3.5-\mathrm{mm}$ irrigated-tip mapping catheter with a 1-mm tip-to-ring interelectrode distance demonstrated that $95 \%$ of unipolar electrograms exhibited a peak-to-peak voltage $>3.8 \mathrm{mV}$ in the right ventricular free wall or $>4.5 \mathrm{mV}$ in the right ventricular septum [37]. In a recent endocardial-epicardial high-density mapping study performed in BrS patients with aborted SCD using a 5-spline multielectrode catheter (20 electrodes with $2-$
6-2 $\mathrm{mm}$ interelectrode spacing), we demonstrated that the $5.3 \mathrm{mV}$ criterion of unipolar endocardial signals displayed $92.4 \%$ specificity and $86.3 \%$ sensitivity for the detection of epicardial bipolar low-voltage areas $<1 \mathrm{mV}$ (Fig. 1). In addition, the previously studied $4 \mathrm{mV}$ cut-off value for unipolar signals displayed $100 \%$ specificity but only $40 \%$ sensitivity for the identification of epicardial lesions [38].

The accuracy of endocardial unipolar EAM to identify distant epicardial scar is compromised by several factors, including tissue thickness, the electrode orientation with respect to the tissue, the presence of endocardial scar (low bipolar voltage areas), the electrode size of the mapping catheter, the tissue contact, and the number of sampling points (high-density) [16]. Therefore, the exact "field of view" of unipolar recordings is not completely predictable. A compact area of unipolar voltage abnormalities possibly reflects real anatomic abnormalities deeper into the endocardial layer [3]. By lowering the unipolar voltage slider bar down (from 5 to 4 or $3 \mathrm{mV}$ ) and still demonstrating abnormalities, an intramural and/or epicardial scar is suggested with a higher degree of certainty (Fig. 2).

\section{Endocardial unipolar voltage abnormalities predict ventricular fibrillation inducibility during programmed ventricular stimulation}

The prognostic significance of VF inducibility during PVS is under continuous debate. There are numerous reasons for the discrepancy of data regarding PVS [16]. First, methodological differences in the stimulation protocols, including the number of extra stimuli, the minimum coupling interval used, and the stimulation site, have a significant impact on PVS results [39]. Second, the inducibility rate may be correlated to the BrS ECG type on the day of the procedure [40]. Third, data regarding the reproducibility of PVS are very limited and do not correlate with clinical presentation [41]. Finally, a false positive VF induction cannot be ruled out.

Endocardial unipolar EAM may improve the prognostic accuracy with respect to true positive PVS results in BrS. We have initially tested the hypothesis that RVOT abnormalities detected by high-density endocardial unipolar EAM mapping predict VF inducibility during PVS [42]. The study population consisted of 17 asymptomatic probands with spontaneous type 1 BrS ECG pattern referred for risk stratification with PVS. A comprehensive evaluation including late gadolinium enhancement cardiac MRI ruled out structural heart disease in all patients. An EAM was considered abnormal in the presence of low-voltage areas $>1.5 \mathrm{~cm}^{2}$ including $\geq 3$ adjacent points with a unipolar signal amplitude $<4 \mathrm{mV}$. PVS induced VF in 6 patients (35\%). Patients with VF inducibility demonstrated greater areas of abnormal unipolar signals $\left(16.0 \pm 3.8 \mathrm{~cm}^{2}\right.$ vs. 8.1 $\pm 4.0 \mathrm{~cm}^{2}$ ) compared with those without arrhythmia induction. Receiver operating characteristics curve analysis 


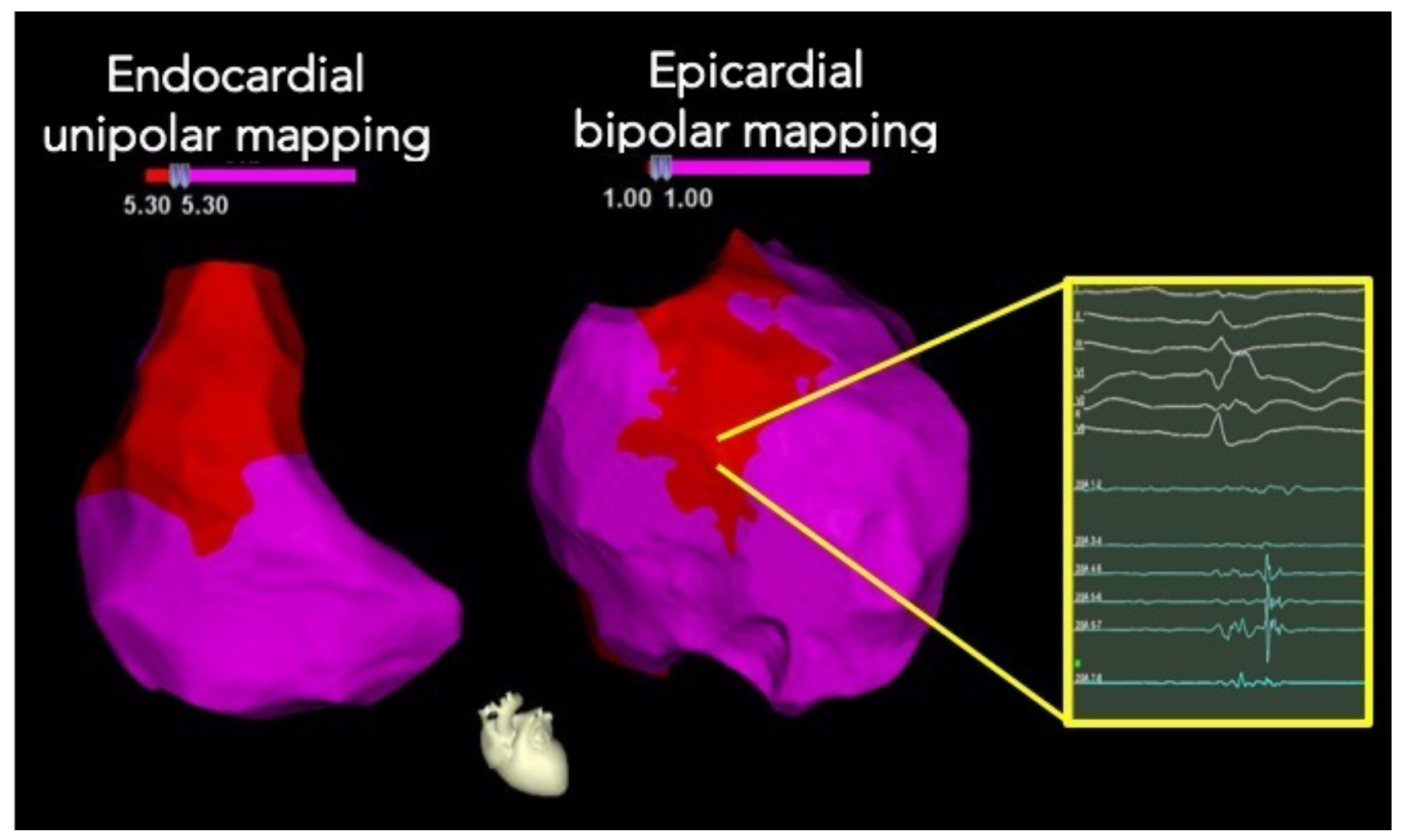

Fig. 1. Combined endocardial-epicardial RV/RVOT high density electroanatomical mapping in BrS. Endocardial unipolar voltage abnormalities $(<5.3 \mathrm{mV})$ correlate with epicardial bipolar voltage abnormalities $(<1 \mathrm{mV})$. Abnormal long duration fractionated bipolar electrograms are recorded at the epicardial aspect of the RVOT. Abbreviations: BrS, Brugada syndrome; EGM, electrogram; RV/RVOT, right ventricle/right ventricular outflow tract.

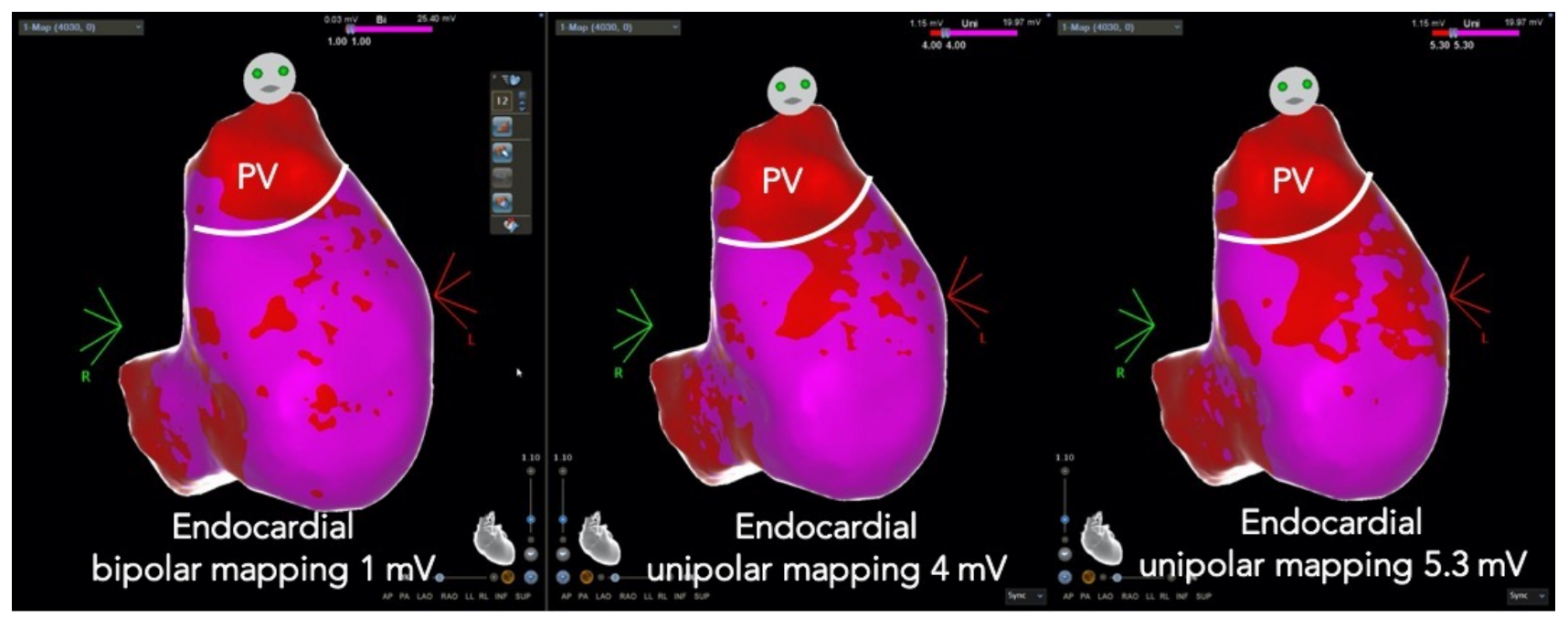

Fig. 2. High density electroanatomical mapping showing patchy areas of low-voltage bipolar signals $(<1 \mathrm{mV})$ at the free wall of the RVOT (modified right anterior oblique view); Large low-voltage unipolar areas are revealed at the RVOT using different unipolar thresholds (4 and $5.3 \mathrm{mV}$ ). By lowering the unipolar voltage slider bar down (from 5.3 to $4 \mathrm{mV}$ ) and still demonstrating abnormalities, an intramural and/or epicardial scar is suggested with a higher degree of certainty. Abbreviations: RVOT, right ventricular outflow tract.

demonstrated that the presence of an area size $>11 \mathrm{~cm}^{2}$ for low-amplitude unipolar signals predict VF inducibility during PVS (sensitivity, 100\%; specificity, 73\%). On the con- trary, subjects with normal endocardial unipolar EAM were non-inducible. Pieroni et al. [11] have elegantly shown that inducible patients display significantly greater endocardial 


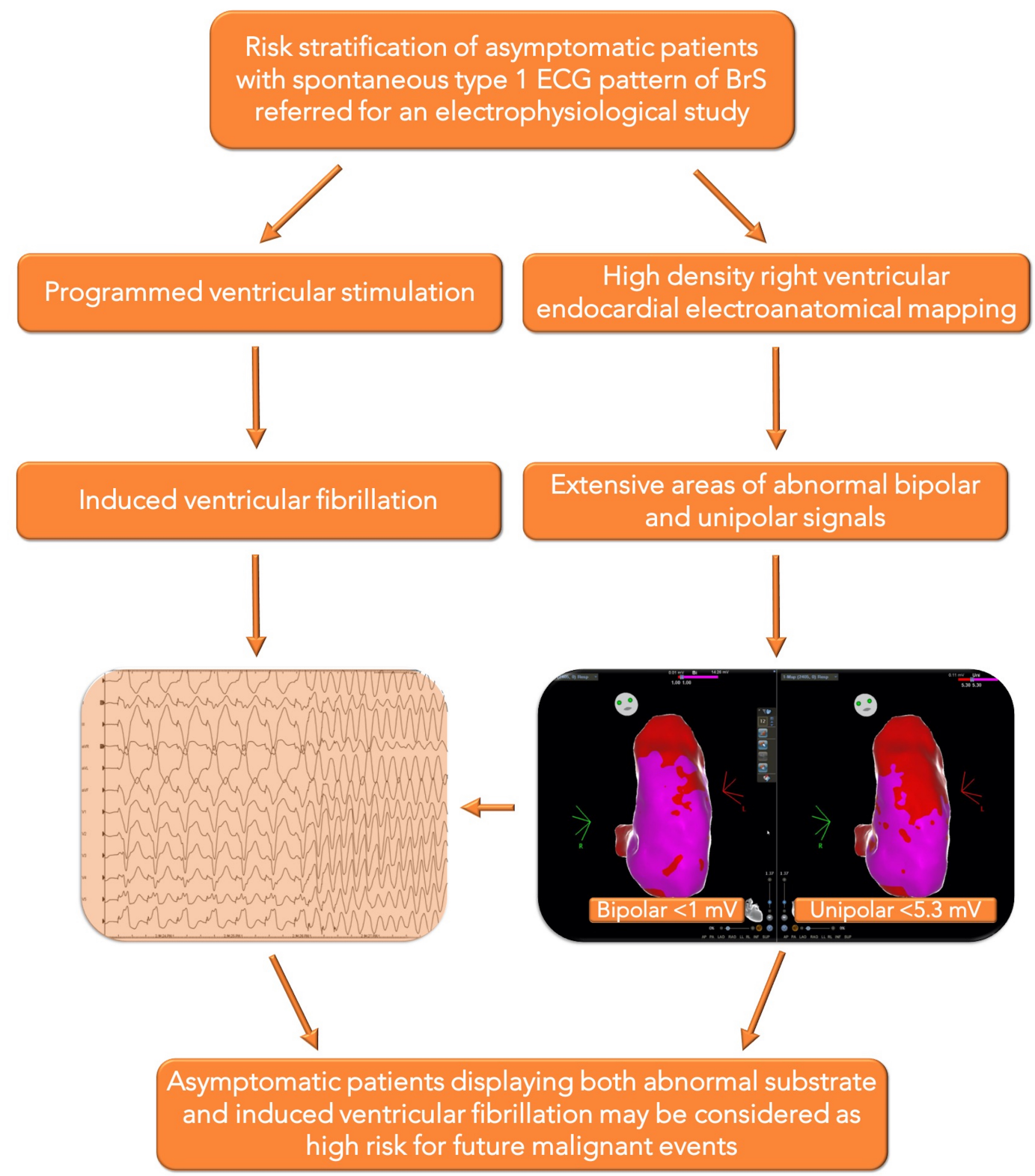

Fig. 3. Proposed workflow for risk stratification of asymptomatic individuals with BrS referred for an electrophysiological study. The presence of abnormal RVOT substrate revealed by high-density EAM predicts VF inducibility and is possibly related to symptom status. Subjects with abnormal substrate and induced VF may be at higher risk for future arrhythmic events. Abbreviations, BrS, Brugada syndrome; EAM, electroanatomical mapping; ECG, electrocardiogram; RVOT, right ventricular outflow tract; VF, ventricular fibrillation.

unipolar low-voltage areas compared with non-inducible ones. These findings have been confirmed in epicardial mapping studies. Pappone et al. [5] have demonstrated that wide epicardial abnormal areas with fragmented longduration ventricular potentials are found in patients with inducible VF compared to patients without inducible arrhythmias, irrespective of clinical presentation. A substrate size of $4 \mathrm{~cm}^{2}$ showed a good performance in identifying patients with inducible arrhythmias. Of note, patients with wider substrates became inducible with a less aggressive protocol.

\section{Endocardial unipolar voltage abnormalities as a risk stratification tool in Brugada syndrome}

Risk stratification of BrS patients still remains puzzling and represents a great challenge. The prognostic significance of spontaneous type-1 ECG pattern, QRS fragmentation, family history of SCD, VF inducibility during PVS, sinus node dysfunction, and even syncope merit special consideration as single risk factors before implanting an ICD [16]. Multiparametric scores, including the markers mentioned above, have been introduced into clinical prac- 
tice for a better selection approach $[18,19]$. However, novel markers are mandatory to improve risk stratification of $\mathrm{BrS}$ patients, especially of asymptomatic ones.

In a recent study including 14 patients with $\mathrm{BrS}$ and aborted SCD in the setting of documented VF and 40 asymptomatic individuals with spontaneous type-1 ECG patterns, we evaluated the prognostic significance of RVOT electroanatomical abnormalities [38]. A combined highdensity endocardial-epicardial mapping procedure was performed in all cases with aborted SCD. All symptomatic patients with aborted SCD displayed abnormal endocardialepicardial maps. The endocardial unipolar low-voltage areas $(<5.3 \mathrm{mV})$ mainly located at the anterior aspect of the RVOT were colocalized with the epicardial bipolar lowvoltage areas $(<1 \mathrm{mV})$ in all subjects. Patients with aborted SCD exhibited significantly wider endocardial unipolar and bipolar low-voltage areas in relation to asymptomatic patients. The presence of endocardial unipolar low-voltage areas $>14.5 \mathrm{~cm}^{2}$ discriminated symptomatic from asymptomatic individuals with a sensitivity of $92.5 \%$ and a specificity of $72.5 \%$ [38]. Using the strict $4 \mathrm{mV}$ criterion for unipolar signals, the difference in unipolar low-voltage areas between symptomatic and asymptomatic individuals remained statistically significant.

In $\mathrm{BrS}$, the presence of both repolarization and depolarization abnormalities constitute the functional and anatomical substrates that are possibly implicated in VF maintenance [17]. The mechanisms that maintain VF are partially clarified. The presence of arrhythmogenic substrates related to structural abnormalities is considered a key factor for sustained VF [43,44]. Fibrillatory mechanisms are strongly related to the underlying electro-architectural substrate including fibrosis patterns and the degree of abnormal gap junction coupling [44]. VF is sustained by a continuous spectrum of mechanisms ranging from organized fibrillation sustained by stable rotational activities to disorganized fibrillation without stable rotational activities [44]. Previous studies have demonstrated that rotational activities are mainly localized to areas of greater fibrosis in perfused cardiomyopathic hearts and low voltage areas during EAM $[45,46]$. Based on these findings, the extent of the abnormal substrate may play an essential role in VF maintenance in BrS. A proposed workflow for risk stratification of asymptomatic individuals with $\mathrm{BrS}$ referred for an electrophysiological study is shown in the Fig. 3. Subjects displaying both abnormal RVOT substrate and induced VF may be at higher risk for future arrhythmic events.

\section{Conclusions}

Preliminary findings correlate the presence of largesized electroanatomical abnormalities with both VF inducibility during PVS and/or symptom status in patients BrS. Due to the certain limitations of PVS, the identification of microstructural alterations in BrS by different imag- ing modalities, including endocardial unipolar EAM, may assist in the risk stratification of patients with BrS.

\section{Author contributions}

KPL - conception of the idea, wrote the first draft, critical revisions, final approval; KV, ME, SD, PK, GT, TL, GB, PN, EP, FS, MH, AB — critical revision, final approval.

\section{Ethics approval and consent to participate}

Not applicable.

\section{Acknowledgment}

Not applicable.

\section{Funding}

This research received no external funding.

\section{Conflict of interest}

The authors declare no conflict of interest. Konstantinos P. Letsas is serving as one of the Guest editors of this journal. We declare that Konstantinos P. Letsas had no involvement in the peer review of this article and has no access to information regarding its peer review. Full responsibility for the editorial process for this article was delegated to Peter A. McCullough.

\section{References}

[1] Sieira J, Brugada P. The definition of the Brugada syndrome. European Heart Journal. 2017; 38: 3029-3034.

[2] Martini B, Nava A, Thiene G, Buja GF, Canciani B, Scognamiglio $\mathrm{R}$, et al. Ventricular fibrillation without apparent heart disease: description of six cases. American Heart Journal. 189; 118: 1203-1209.

[3] Frustaci A, Priori SG, Pieroni M, Chimenti C, Napolitano C, Rivolta I, et al. Cardiac histological substrate in patients with clinical phenotype of Brugada syndrome. Circulation. 2005; 112: 3680-3687.

[4] Nademanee K, Raju H, de Noronha SV, Papadakis M, Robinson L, Rothery S, et al. Fibrosis, Connexin-43, and Conduction Abnormalities in the Brugada Syndrome. Journal of the American College of Cardiology. 2015; 66: 1976-1986.

[5] Pappone C, Ciconte G, Manguso F, Vicedomini G, Mecarocci $\mathrm{V}$, Conti M, et al. Assessing the Malignant Ventricular Arrhythmic Substrate in Patients with Brugada Syndrome. Journal of the American College of Cardiology. 2018; 71: 1631-1646.

[6] Antzelevitch C. Genetic, molecular and cellular mechanisms underlying the J wave syndromes. Circulation Journal. 2012; 76: 1054-1065.

[7] Watanabe H, Minamino T. Genetics of Brugada syndrome. Journal of Human Genetics. 2016; 61: 57-60.

[8] Skinner JR, Love DR. The SCN5A gene in Brugada syndrome: mutations, variants, missense and nonsense. What's a clinician to do? Heart Rhythm. 2010; 7: 50-51.

[9] Tse G, Wong ST, Tse V, Yeo JM. Depolarization vs. repolarization: what is the mechanism of ventricular arrhythmogenesis underlying sodium channel haploinsufficiency in mouse hearts? Acta Physiologica. 2016; 218: 234-235.

[10] Tse G, Liu T, Li KHC, Laxton V, Chan YWF, Keung W, et al. Electrophysiological Mechanisms of Brugada Syndrome: In- 
sights from Pre-clinical and Clinical Studies. Frontiers in Physiology. 2016; 7: 467.

[11] Pieroni M, Notarstefano P, Oliva A, Campuzano O, Santangeli $\mathrm{P}$, Coll M, et al. Electroanatomic and Pathologic Right Ventricular Outflow Tract Abnormalities in Patients with Brugada Syndrome. Journal of the American College of Cardiology. 2018; 72: $2747-2757$.

[12] Miles C, Asimaki A, Ster IC, Papadakis M, Gray B, Westaby J, et al. Biventricular Myocardial Fibrosis and Sudden Death in Patients with Brugada Syndrome. Journal of the American College of Cardiology. 2021; 78: 1511-1521.

[13] Nademanee K, Hocini M, Haïssaguerre M. Epicardial substrate ablation for Brugada syndrome. Heart Rhythm. 2017; 14: 457461.

[14] Pappone C, Brugada J, Vicedomini G, Ciconte G, Manguso F, Saviano M, et al. Electrical Substrate Elimination in 135 Consecutive Patients with Brugada Syndrome. Circulation. Arrhythmia and Electrophysiology. 2017; 10: e005053.

[15] Pannone L, Monaco C, Sorgente A, Vergara P, Calburean P, Gauthey A, et al. High-density epicardial mapping in Brugada syndrome: Depolarization and repolarization abnormalities. Heart Rhythm. 2021. (in press)

[16] Bazan V, Frankel DS, Santangeli P, Garcia FC, Tschabrunn CM, Marchlinski FE. Three-dimensional myocardial scar characterization from the endocardium: Usefulness of endocardial unipolar electroanatomic mapping. Journal of Cardiovascular Electrophysiology. 2019; 30: 427-437.

[17] Letsas KP, Asvestas D, Baranchuk A, Liu T, Georgopoulos S, Efremidis M, et al. Prognosis, risk stratification, and management of asymptomatic individuals with Brugada syndrome: a systematic review. Pacing and Clinical Electrophysiology. 2017; 40: 1332-1345.

[18] Letsas KP, Bazoukis G, Efremidis M, Georgopoulos S, Korantzopoulos $\mathrm{P}$, Fragakis N, et al. Clinical characteristics and longterm clinical course of patients with Brugada syndrome without previous cardiac arrest: a multiparametric risk stratification approach. Europace. 2019; 21: 1911-1918.

[19] Sieira J, Conte G, Ciconte G, Chierchia G, Casado-Arroyo R, Baltogiannis $\mathrm{G}$, et al. A score model to predict risk of events in patients with Brugada Syndrome. European Heart Journal. 2017; 38: $1756-1763$.

[20] Zumhagen S, Spieker T, Rolinck J, Baba HA, Breithardt G, Böcker W, et al. Absence of Pathognomonic or Inflammatory Patterns in Cardiac Biopsies from Patients with Brugada Syndrome. Circulation: Arrhythmia and Electrophysiology. 2009; 2: 16-23.

[21] Ohkubo K, Watanabe I, Okumura Y, Takagi Y, Ashino S, Kofune $\mathrm{M}$, et al. Right ventricular histological substrate and conduction delay in patients with Brugada syndrome. International Heart Journal. 2010; 51: 17-23.

[22] Takagi M, Aihara N, Kuribayashi S, Taguchi A, Shimizu W, Kurita $\mathrm{T}$, et al. Localized right ventricular morphological abnormalities detected by electron-beam computed tomography represent arrhythmogenic substrates in patients with the Brugada syndrome. European Heart Journal. 2001; 22: 1032-1041.

[23] Catalano O, Antonaci S, Moro G, Mussida M, Frascaroli M, Baldi $\mathrm{M}$, et al. Magnetic resonance investigations in Brugada syndrome reveal unexpectedly high rate of structural abnormalities. European Heart Journal. 2009; 30: 2241-2248.

[24] Bastiaenen R, Cox AT, Castelletti S, Wijeyeratne YD, Colbeck $\mathrm{N}$, Pakroo $\mathrm{N}$, et al. Late gadolinium enhancement in Brugada syndrome: a marker for subtle underlying cardiomyopathy? Heart Rhythm. 2017; 14: 583-589.

[25] Pappone C, Mecarocci V, Manguso F, Ciconte G, Vicedomini G, Sturla F, et al. New electromechanical substrate abnormalities in high-risk patients with Brugada syndrome. Heart Rhythm. 2020;
17: $637-645$.

[26] Nademanee K, Veerakul G, Chandanamattha P, Chaothawee L, Ariyachaipanich A, Jirasirirojanakorn K, et al. Prevention of ventricular fibrillation episodes in Brugada syndrome by catheter ablation over the anterior right ventricular outflow tract epicardium. Circulation. 2011; 123: 1270-1279.

[27] Zhang P, Tung R, Zhang Z, Sheng X, Liu Q, Jiang R, et al. Characterization of the epicardial substrate for catheter ablation of Brugada syndrome. Heart Rhythm. 2016; 13: 2151-2158.

[28] Letsas KP, Efremidis M, Vlachos K, Georgopoulos S, Karamichalakis N, Asvestas D, et al. Right ventricular outflow tract high-density endocardial unipolar voltage mapping in patients with Brugada syndrome: evidence for electroanatomical abnormalities. Europace. 2018; 20: f57-f63.

[29] Lambiase PD, Ahmed AK, Ciaccio EJ, Brugada R, Lizotte E, Chaubey S, et al. High-Density Substrate Mapping in Brugada Syndrome: combined role of conduction and repolarization heterogeneities in arrhythmogenesis. Circulation. 2009; 120: 106117.

[30] Postema PG, van Dessel PFHM, de Bakker JMT, Dekker LRC, Linnenbank AC, Hoogendijk MG, et al. Slow and Discontinuous Conduction Conspire in Brugada Syndrome. Circulation: Arrhythmia and Electrophysiology. 2008; 1: 379-386.

[31] Agullo-Pascual E, Cerrone M, Delmar M. Arrhythmogenic cardiomyopathy and Brugada syndrome: Diseases of the connexome. FEBS Letters. 2014; 588: 1322-1330.

[32] Cerrone M, Delmar M. Desmosomes and the sodium channel complex: implications for arrhythmogenic cardiomyopathy and Brugada syndrome. Trends in Cardiovascular Medicine. 2014; 24: 184-190.

[33] Polin GM, Haqqani H, Tzou W, Hutchinson MD, Garcia FC, Callans DJ, et al. Endocardial unipolar voltage mapping to identify epicardial substrate in arrhythmogenic right ventricular cardiomyopathy/dysplasia. Heart Rhythm. 2011; 8: 76-83.

[34] Tokuda M, Tedrow UB, Inada K, Reichlin T, Michaud GF, John $\mathrm{RM}$, et al. Direct comparison of adjacent endocardial and epicardial electrograms: implications for substrate mapping. Journal of the American Heart Association. 2013; 2: e000215.

[35] Venlet J, Piers SRD, Kapel GFL, de Riva M, Pauli PFG, van der Geest RJ, et al. Unipolar Endocardial Voltage Mapping in the Right Ventricle: Optimal Cutoff Values Correcting for Computed Tomography-Derived Epicardial Fat Thickness and their Clinical Value for Substrate Delineation. Circulation: Arrhythmia and Electrophysiology. 2017; 10: e005175.

[36] Chrispin J, Keramati AR, Assis FR, Misra S, Zghaib T, Berger $\mathrm{RD}$, et al. Correlation of right ventricular multielectrode endocardial unipolar mapping and epicardial scar. Pacing and Clinical Electrophysiology. 2018; 41: 345-352.

[37] Lee AC, Strugnell W, Vittinghoff E, Hamilton-Craig C, Haqqani HM. Right Ventricular Electrogram Characteristics in a T1 Mapping-Validated Normal Population: Implications for Unipolar Voltage Mapping. JACC: Clinical Electrophysiology. 2020; 6: 711-721.

[38] Letsas KP, Vlachos K, Conte G, Efremidis M, Nakashima $\mathrm{T}$, Duchateau J, et al. Right ventricular outflow tract electroanatomical abnormalities in asymptomatic and high-risk symptomatic patients with Brugada syndrome: Evidence for a new risk stratification tool? Journal of Cardiovascular Electrophysiology. 2021; 32: 2997-3007.

[39] Eckardt L, Kirchhof P, Schulze-Bahr E, Rolf S, Ribbing M, Loh $\mathrm{P}$, et al. Electrophysiologic investigation in Brugada syndrome; yield of programmed ventricular stimulation at two ventricular sites with up to three premature beats. European Heart Journal. 2002; 23: 1394-1401.

[40] Probst V, Veltmann C, Eckardt L, Meregalli PG, Gaita F, Tan $\mathrm{HL}$, et al. Long-term prognosis of patients diagnosed with Bru- 
gada syndrome: Results from the FINGER Brugada Syndrome Registry. Circulation. 2010; 121: 635-643.

[41] Sieira J, Conte G, Ciconte G, de Asmundis C, Chierchia G, Baltogiannis G, et al. Prognostic Value of Programmed Electrical Stimulation in Brugada Syndrome: 20 years experience. Circulation: Arrhythmia and Electrophysiology. 2015; 8: 777-784.

[42] Letsas KP, Efremidis M, Asvestas D, Vlachos K, Georgopoulos $\mathrm{S}$, Tse G, et al. Right Ventricular Outflow Tract Electroanatomical Abnormalities Predict Ventricular Fibrillation Inducibility in Brugada Syndrome. Circulation. Arrhythmia and Electrophysiology. 2018; 11: e005928.

[43] Haïssaguerre M, Duchateau J, Dubois R, Hocini M, Cheniti G, Sacher F, et al. Idiopathic Ventricular Fibrillation: Role of Purkinje System and Microstructural Myocardial Abnormalities. JACC: Clinical Electrophysiology. 2020; 6: 591-608.
[44] Handa BS, Li X, Baxan N, Roney CH, Shchendrygina A, Mansfield CA, et al. Ventricular fibrillation mechanism and global fibrillatory organization are determined by gap junction coupling and fibrosis pattern. Cardiovascular Research. 2021; 117: 10781090.

[45] Nair K, Umapathy K, Farid T, Masse S, Mueller E, Sivanandan $\mathrm{RV}$, et al. Intramural activation during early human ventricular fibrillation. Circulation. Arrhythmia and Electrophysiology. 2011; 4: 692-703.

[46] Massé S, Downar E, Chauhan V, Sevaptsidis E, Nanthakumar K. Ventricular fibrillation in myopathic human hearts: mechanistic insights from in vivo global endocardial and epicardial mapping. American Journal of Physiology-Heart and Circulatory Physiology. 2007; 292: H2589-H2597. 